\title{
Enhanced Production of Microbial Metabolites in the Presence of Dimethyl Sulfoxide
}

\author{
Genhui Chen, Gui-Yang-Sheng Wang, Xiang Li, \\ Barbara Waters and Julian Davies* \\ TerraGen Discovery Inc. \\ Suite 300-2386 East Mall-UBC, Vancouver, B.C. V6T 1Z3, Canada
}

(Received for publication June 26, 2000)

\begin{abstract}
Bacterial strains grown in the presence of low concentrations of dimethyl sulfoxide (DMSO) exhibit significant qualitative and quantitative alterations in the production of secondary metabolites. This effect was confirmed for a variety of biosynthetic families, including chloramphenicol (chorismate), thiostrepton (peptide) and tetracenomycin (polyketide), and for natural and recombinant strains of streptomycetes; a similar effect was seen with antibiotic-producing bacilli such as $B$. circulans. Increase in antibiotic production was not the result of a change in the growth rate of these organisms, since yields of biomass were similar in media with and without DMSO (up to $3 \%$ ). We suggest that the addition of compounds such as DMSO provides a means of examining the full biosynthetic potential of microbes and might be used to promote secondary metabolite production. The mode of action of DMSO is not known, but in the cases studied it may act at the level of translation.
\end{abstract}

Environmental factors affect microbial secondary metabolism. Stress, starvation or supplementation with nutrients such as amino acids, phosphate, nitrogen, etc., have a plethora of effects on the production of secondary metabolites. It has long been standard practice in the pharmaceutical industry to develop specific and often complex culture media to maximize the production yields of secondary metabolites such as antibiotics ${ }^{1,2}$. In addition, a variety of mutants have been isolated that influence the levels and timing of secondary metabolic pathway function, for example, those affecting intracellular cyclic AMP formation $^{3,4)}$. More importantly in an environmental sense, low-molecular-weight signaling agents are known to have profound effects on certain biosynthetic and developmental pathways through ligand-receptor interactions, indicating their important physiological and environmental roles ${ }^{5,6)}$.

Many mutations have been introduced into so-called production strains to enhance the yield of a particular antibiotic and in the majority of cases, the genetic and biochemical nature of these strains is unknown ${ }^{7}$. Recent work by OCHI and co-workers ${ }^{8,9\}}$ showed that mutations to streptomycin resistance dramatically influence the production of polyketides and other antibiotics in
Streptomyces and Bacillus. While the biochemical basis of this effect is not completely understood, there is evidence that ppGpp levels are altered in the streptomycin-resistant mutants and it is known that ppGpp production reaches a miximum at the time when the antibiotics are being made $^{10)}$. The streptomycin-resistant mutations (and variations in ppGpp) are invariably pleiotropic, causing developmental and other phenotypic changes. Distinct streptomycin-resistance alleles (alterations of $r p s L$ ) have different phenotypic effects ${ }^{11)}$.

It has long been known that streptomycin and other aminoglycoside antibiotics disrupt translation in vitro and in vivo in susceptible bacterial strains and that these effects can be modulated by antibiotic resistance alleles ${ }^{12)}$. Interestingly, it has been shown that some of the consequences of the perturbation of translation by streptomycin (phenotypic suppression, for example) can be mimicked by the addition of organic compounds such as ethanol or dimethyl sulfoxide (DMSO) to growing cultures. For example, the addition of low concentrations of these molecules $(1 \sim 3 \%)$ to cell-free systems causes mistranslation ${ }^{13,14)}$ and even permits the growth of streptomycin-dependent mutants on culture medium in the 
absence of the antibiotic. The original and most marked phenotypic effect of streptomycin addition was the suppression of nonsense mutants in amino acid biosynthetic pathways, and this result could be duplicated by the addition of low concentrations of ethanol or $\mathrm{DMSO}^{15)}$.

The similarity of the effects of streptomycin-resistance mutations on secondary metabolite formation, as demonstrated by $\mathrm{OCHI}^{8,9)}$, and the earlier demonstrations of solvent-induced aberrations on translation prompted us to test the effects of organic compounds on secondary metabolite production by antibiotic-producing organisms, and we report here that significant metabolic variation is caused by the addition of low concentrations (1 $2 \%$ ) of DMSO to growing cultures of streptomycetes and other bacteria. In addition to alterations in morphology, such as spore formation and pigment production, the growth of antibiotic-producing organisms in the presence of low concentrations of organic compounds results in qualitative and quantitative changes in secondary metabolite production. This could have value in natural product drugdiscovery programmes, since solvent-induced effects may reveal the presence of secondary metabolites that are otherwise present in low concentrations under normal growth conditions.

\section{Materials and Methods}

\section{Culture Conditions}

Streptomyces strains from various sources were grown on solid ISP4 medium (Difco) until sporulation. The spores were harvested by washing the plate surface with sterile water. This spore suspension was mixed with an equal amount of $50 \%$ glycerol and stored at $-30^{\circ} \mathrm{C}$ for future use. Culture media were initially inoculated with a spore suspension and mixed thoroughly; $10 \mathrm{ml}$ of inoculated medium was transferred into each of four $50-\mathrm{ml}$ shake tubes. The organic compounds (DMSO, ethanol, dimethyl sulfone, etc.) were added to the medium at the indicated concentrations before addition of spores. The tubes were plugged with a sterile sponge and incubated at $30^{\circ} \mathrm{C}$ with shaking $(180 \mathrm{rpm})$. Bacillus strains were inoculated from colonies on tryptic soy agar into tryptic soy broth and grown with shaking at $30^{\circ}$ with or without supplementation.

\section{Preparation of Samples for Chromatographic Analysis}

One $\mathrm{ml}$ of culture was removed and mixed by shaking (450 rpm, 30 minutes) with an equal volume of ethyl acetate in a 2-ml Eppendorf tube. Following centrifugation
(14,000 rpm, 5 minutes), $0.75 \mathrm{ml}$ of the organic fraction was transferred to a 1-ml Eppendorf tube and dried (Speedivac) at medium heat. The dried residue was dissolved in anhydrous methanol and analyzed by HPLC (Beckman System Gold) and LC/MS. LC/MS chromatograms were obtained on a Hewlett-Packard series $1100 \mathrm{MSD}$ with reversed phase Zorbax $\mathrm{C}_{18}$ rapid column $(2.1 \times 15 \mathrm{~mm})$. The column was eluted with a linear gradient of methanol and $0.5 \%$ formic acid-water, starting from $25 \%$ to $100 \% \mathrm{CH}_{3} \mathrm{OH}$ in 3.5 minutes and $100 \%$ $\mathrm{CH}_{3} \mathrm{OH}$ for 2.5 minutes.

\section{Measurement of Growth}

The growth of streptomycetes was measured by determination of cell biomass. Liquid cultures were centrifuged $(4,000 \mathrm{rpm}, 10$ minutes), the supernatant removed, and the pellet washed twice by resuspending in $40 \mu \mathrm{l}$ of distilled water. The cells were harvested by filtering the cell suspension through a \#1 Whatman filter paper. The filter paper with the cells was then baked in an oven at $80^{\circ} \mathrm{C}$ overnight and weighed.

\section{Morphological Observations}

Stock Streptomyces were first grown in liquid R5 for $24 \sim 48$ hours, and $0.1 \mathrm{ml}$ of the actively growing cell suspension was transferred to solid R5 medium $^{16)}$ with or without DMSO. The plates were incubated at $30^{\circ} \mathrm{C}$ and differences in colony appearance, sporulation and pigmentation were recorded after incubation for $6 \sim 10$ days, depending on species.

\section{Tetracenomycin Production}

Streptomyces glaucescens was used for the production of tretracenomycin in this study. The culture and extraction procedure was the same as outlined above except that $10 \mu 1$ of $1 \mathrm{M} \mathrm{KH}_{2} \mathrm{PO}_{4}$ was added to each $1 \mathrm{ml}$ of culture sample immediately before extraction with ethyl acetate (HPLC grade). Analysis of secondary metabolites was done using a Beckman System Gold HPLC with a reversed phase $\mathrm{C}_{18}$ column. Standard curves were developed using tetracenomycin purified from $S$. glaucescens ${ }^{17)}$; the pure compound was dissolved in $\mathrm{MeOH}$ and submitted to HPLC developed with a gradient of $\mathrm{H}_{2} \mathrm{O}$ and $\mathrm{CH}_{3} \mathrm{CN}$ from $10: 90$ to $80: 20$ during 20 minutes. Sample concentrations were analyzed as described for the standard curve.

\section{Chloramphenicol Production}

Streptomyces venezuelae strain ATCC10712 was grown in liquid GNY medium ${ }^{18)}$. Culture samples were extracted with an equal volume of ethyl acetate and analyzed by 
HPLC. Standard curves for chloramphenicol concentrations were established using a Zorbax $\mathrm{SB} \mathrm{C}_{18}$ rapid column $(4.6 \times 15 \mathrm{~mm})$ with a gradient of $\mathrm{H}_{2} \mathrm{O}: \mathrm{CH}_{3} \mathrm{CN}$ from $80: 20$ to $0: 100$ in five minutes $(1 \mathrm{ml} /$ minute). Chloramphenicol (Sigma) dissolved in $1: 1 \mathrm{EtOH}$ and water was used as reference.

\section{Thiostrepton Production}

Streptomyces azureus (ATCC14921) was used as production strain. The culture was grown in R5 medium and prepared for analysis as outlined above, except that chloroform was used for extraction. A standard concentration curve was developed using thiostrepton (Sigma). Thiostrepton samples were first dissolved in THF and analyzed by HPLC, using a $\mathrm{C}_{8}$ reversed phase column (Beckman, ultrasphere octyl) with an isocratic mobile phase of $\mathrm{H}_{2} \mathrm{O}-\mathrm{THF}-\mathrm{CH}_{3} \mathrm{OH}(2: 2: 1)$, and monitored at $245 \mathrm{~nm}$.

Secondary Metabolite Production by Streptomyces lividans Transformants

Seven randomly selected transformants were taken from two soil libraries constructed in an S. lividans (TK24) host with the vectors pOJ $446^{19)}$ and pWHM601 ${ }^{20)}$ respectively. These recombinants showed different patterns of secondary metabolite production in different media. Ten $\mathrm{ml}$ of ATCC172 medium was inoculated with $0.2 \mathrm{ml} 5$-day-old mycelium in a 50-ml tube and supplemented with appropriate concentrations of DMSO ( $\mathrm{v} / \mathrm{v}$ ) (see results); ethyl acetate extracts were prepared after five days as described above and analysed by TLC and HPLC. The production of antibiotics was bioassayed against sensitive bacterial indicator strains.

\section{Antibiotic Production by Bacilli}

Bacillus strains grown in tryptic soy broth were extracted with ethyl acetate and analysed by liquid chromatography/ mass spectroscopy.

\section{Results}

\section{Effects of Organic Compounds on Antibiotic} Production by Streptomycetes

A variety of organic compounds (including DMSO, tetrahydrofuran, dimethylformamide, acetonitrile, isopropylalcohol, ethanol, methanol, methylethyl ketone, and dioxane) were tested for their effect on chloramphenicol production by Streptomyces venezuelae. The effects were varied substantially with the nature and concentration of the
Fig. 1. Effect of ethanol on biomass (-) and tetracenomycin production $(\mathrm{O}-\mathrm{O})$ by $S$. glaucescens.

(A) grown in TSB. (B) grown in R5.
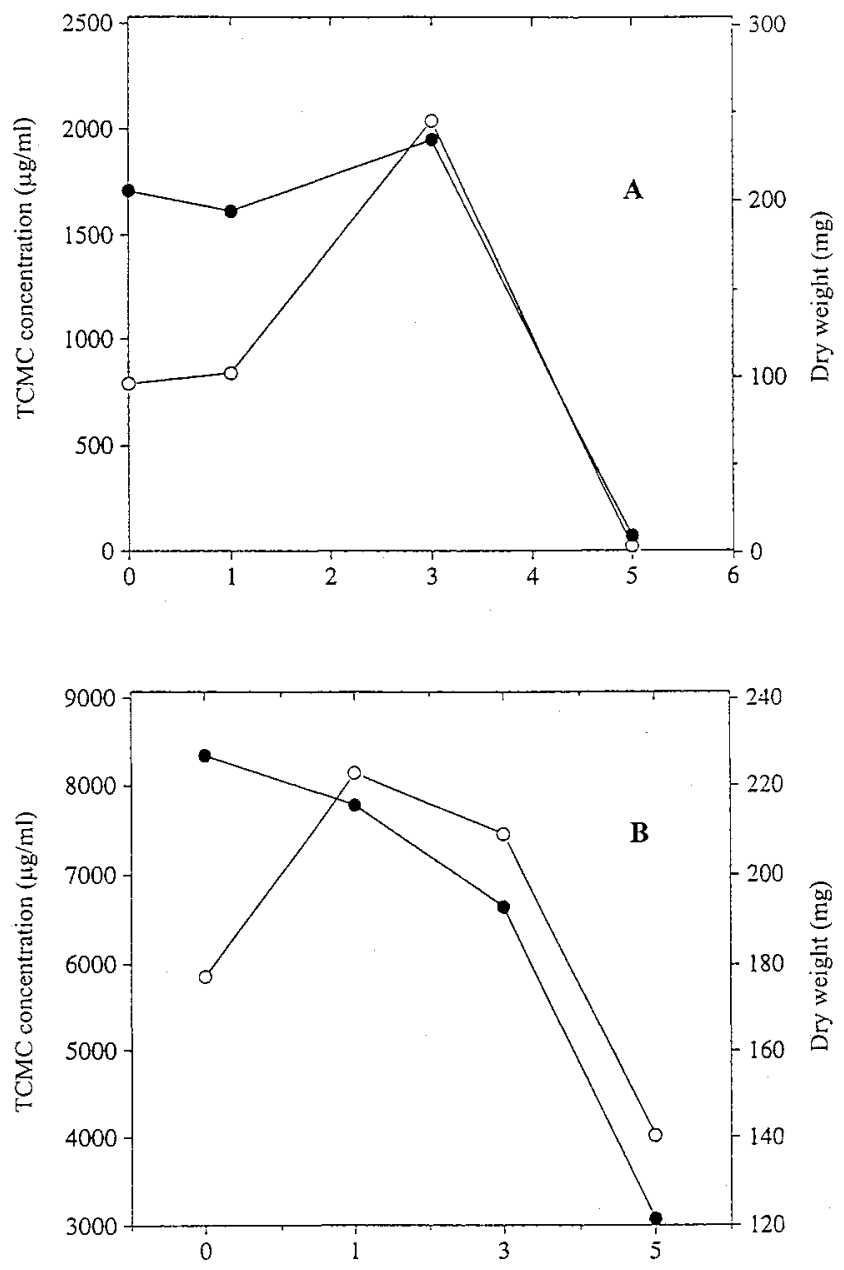

compound added. Most were inhibitory (results not shown) but the presence of dimethyl sulfoxide increased antibiotic production.

The effect of DMSO on the production of chloramphenicol, tetracenomycin, and thiostrepton by their producing organisms was then compared. Enhancement of production was seen in all cases. With $3 \%$ DMSO in the culture media, chloramphenicol and tetracenomycin $\mathrm{C}$ production was increased about three times over the control; the increase in thiostrepton production was about two-fold. The addition of 5\% DMSO had an inhibitory effect on the yield of chloramphenicol but no effect on tetracenomycin $\mathrm{C}$, while thiostrepton production was enhanced (Table 1). 
Table 1. Effect of DMSO on secondary metabolite production by various Streptomyces strains.

\begin{tabular}{|c|c|c|c|}
\hline & \multicolumn{3}{|c|}{ Compound production (mg/g) } \\
\hline DMSO concentration & Chloramphenicol* & Tetracenomycin $\mathrm{C}^{+}$ & Thiostrepton \\
\hline $0 \%$ & $2.5 \pm 0.4$ & $51.6 \pm 6.3$ & $1.7 \pm 0.2$ \\
\hline $1 \%$ & $4.5 \pm 0.8$ & $78.2 \pm 10.3$ & $2.9 \pm 0.3$ \\
\hline $3 \%$ & $8.2 \pm 0.8$ & $124.1 \pm 23.4$ & $2.6 \pm 0.3$ \\
\hline $5 \%$ & $0.2 \pm 0.1$ & $50.9 \pm 18.2$ & $3.2 \pm 0.3$ \\
\hline
\end{tabular}

* S. venezuelae grown in GNY for five days

$\dagger$ S. glaucescens grown in TSB for six days

$\ddagger S$. azureus grown in R5 for five days

\section{Effect of EtOH on Tetracenomycin C}

Ethanol stimulated tetracenomycin $\mathrm{C}$ production in both tryptic soy broth (TSB) and R5 culture media. The addition of $1 \sim 3 \%$ ethanol to these media enhanced production of the antibiotic; the increase was more significant in TSB than in R5 (Fig. 1). EtOH inhibited the growth of $S$. glaucescens at a concentration of $5 \%$ in TSB.

\section{Effect of DMSO on Other Streptomycetes and Bacilli}

DMSO influenced metabolite production and morphology of all wild-type Streptomyces strains tested. $S$. lividans cultures grown on solid medium with $2 \%$ DMSO had earlier and heavier sporulation than those grown on medium without DMSO and produced more pigment in the presence of DMSO (Fig. 2). Substantial differences in HPLC patterns of extracts of liquid cultures supplemented with DMSO were evident in the three strains tested: S. lividans, S. lavendulae and S. rimosus; in the case of $S$. lividans strains LC-MS analysis of extracts from cultures grown with DMSO had significant increases in the production of the pigment prodigiosin (Fig. 3). There were no significant changes in production of biomass when treated and untreated cultures were compared. Similarly, the production of secondary metabolites by antibiotic-producing strains of Bacillus was influenced by the addition of DMSO. Figure 4 shows the effect of $3 \%$
DMSO on metabolite formation by aminoglycosideproducing $B$. circulans and by polymixin-producing $B$. polymyxa as analysed by LC/MS.

\section{Effect on Recombinant Strains of S. lividans}

We were interested in testing the effect of DMSO on secondary metabolite production in recombinant $S$. lividans, with the objective of expanding the range of small molecules produced. The addition of DMSO provoked a variety of effects on $S$. lividans recombinants, depending to some extent on the plasmid present. pOJ446 recombinants were more pigmented in medium with DMSO than without, whereas pWHM601 recombinants had substantially less pigment in medium with DMSO (results not shown). LC-MS patterns of extracts grown in DMSO showed significant qualitative and quantitative variation (Fig. 5), and the antibiotic activity of extracts against bacterial tester strains was also changed by growth in medium containing DMSO.

\section{Effects of Dimethyl Sulphone on Chloramphenicol Production}

A number of bacterial species reduce DMSO through the action of dimethyl sulphide reductase ${ }^{21)}$. Dimethyl sulphone, dimethyl sulphide, and dimethyl sulphoxide were 
Fig. 2. S. lividans TK21 grown on solid R5 medium with (left) and without (right) $3 \%$ DMSO. A-seen from top of petri plate. B-seen from bottom.

$\mathrm{A}$

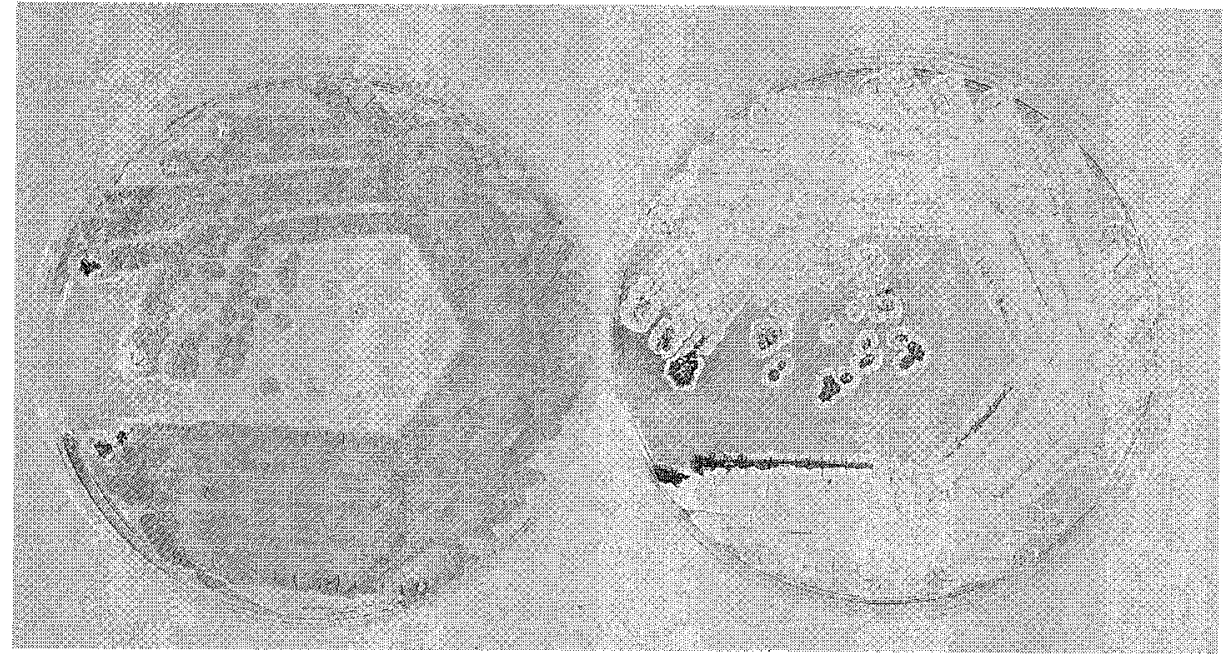

B

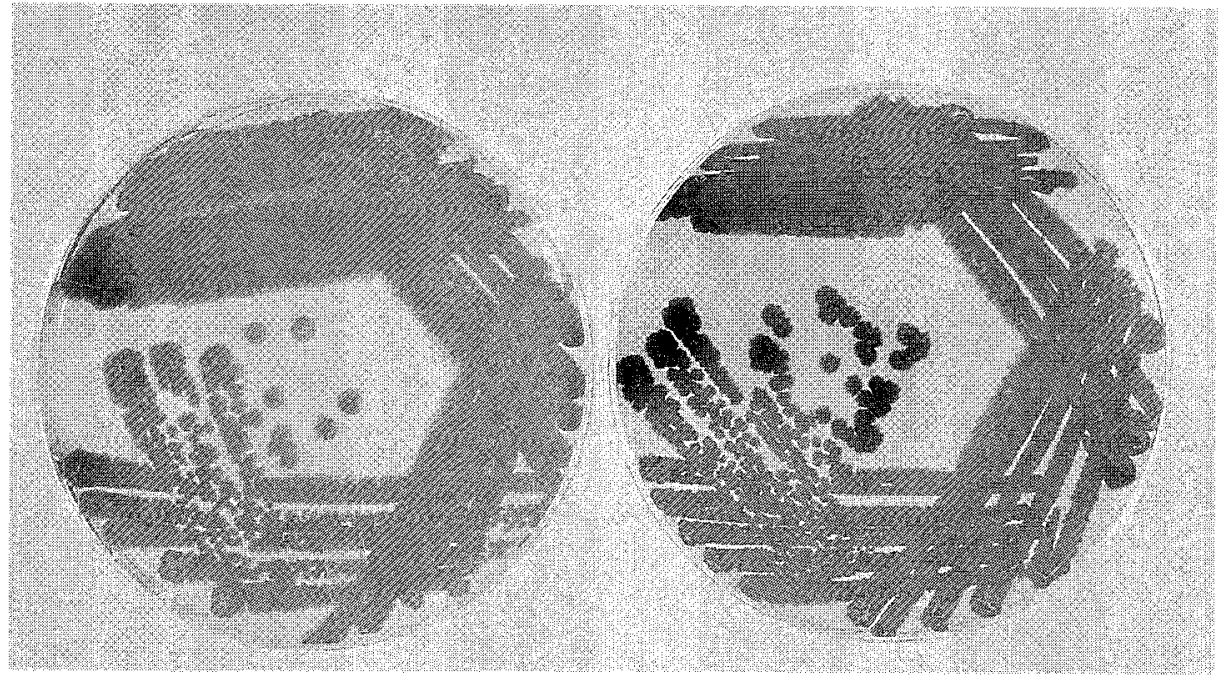

tested for effects on antibiotic production, but only dimethyl sulphone (DMSN) was active, although to a lesser extent than DMSO; typically the addition of $3 \%$ DMSN to cultures of $S$. venezuelae resulted in a $75 \%$ increase in chloramphenicol production (results not shown).

\section{Discussion}

In this study we demonstrate that bacterial strains grown in the presence of low concentrations of DMSO exhibit significant alterations in the production of secondary metabolites. This was demonstrated for a variety of biosynthetic families, including chloramphenicol (chorismate), thiostrepton (peptide) and tetracenomycin (polyketide), and for natural and recombinant strains of streptomycetes. Similar effects were seen with antibioticproducing strains of Bacillus. The increase was not due to changes in growth rate, since yields of biomass were similar in media with and without DMSO (up to $3 \%$ ). However, increasing the DMSO concentration to $5 \%$ or more usually caused significant growth restriction. There was no effect of DMSO on the extraction or solubilisation of products (results not shown). 
Fig. 3. Prodigiosin production by $S$. lividans strains in the absence and presence of $3 \%$ DMSO.
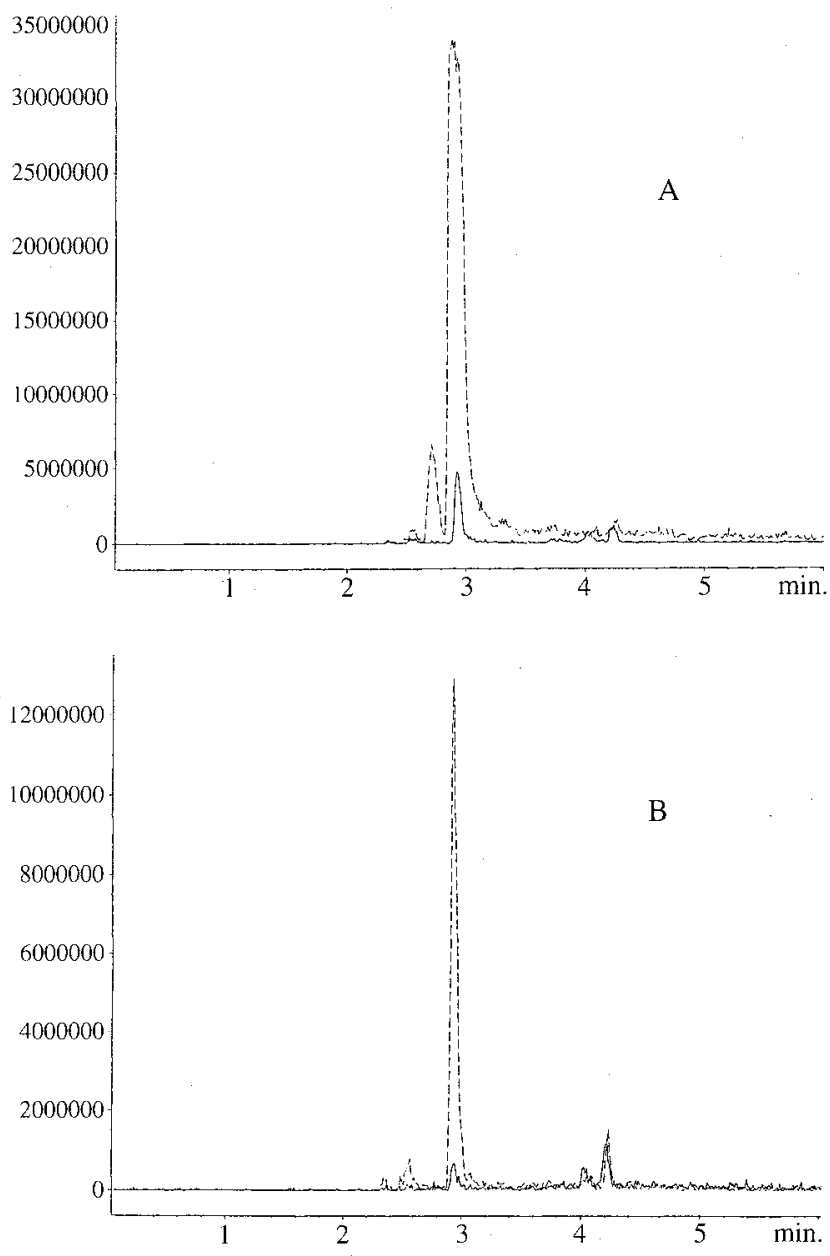

(A) TK21, (B) TK23. Ethyl acetate extracts were analysed by LC/MS as described in Materials and Methods. (Solid line, no DMSO; dotted line, added DMSO).

In recent years there has been significant interest in the cloning of antibiotic biosynthetic genes with heterologous hosts in efforts to generate new types of bioactive metabolites. The hosts employed are usually S. lividans or $S$. coelicolor. The recombinant strains of $S$. lividans used in our studies carried plasmids with inserted DNA fragments isolated from environmental sources (such as soil). In these strains introduction (and presumably expression) of the heterologous DNA caused changes in the secondary metabolite profiles of the recombinants (unpublished observation). In the presence of low concentrations of DMSO $(1 \sim 3 \%)$ the metabolic profiles were substantially altered, and in some cases antibiotic productivity was increased.
Fig. 4. Chromatograms of $B$. circulans (top) and $B$. polymyxa (bottom) in the absence and presence of $3 \%$ DMSO.
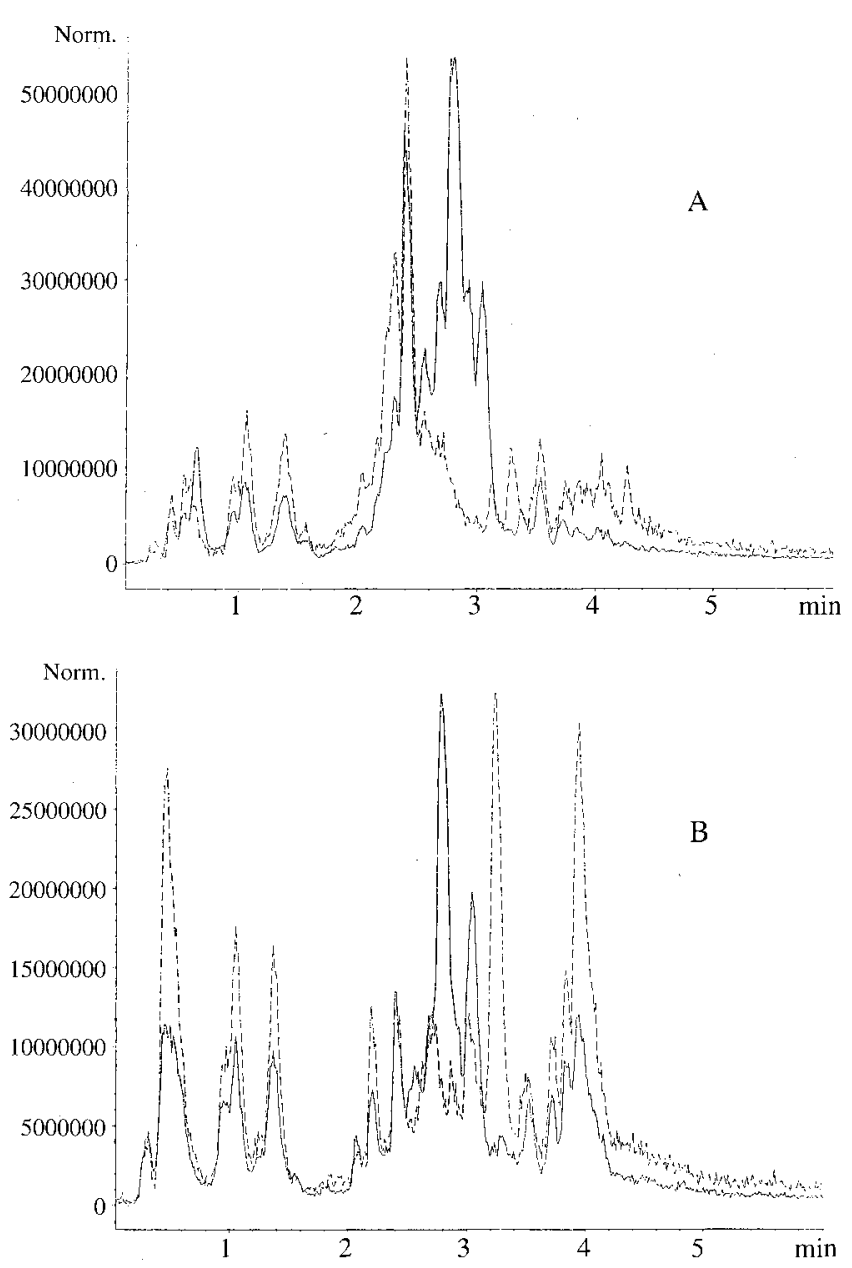

Ethyl acetate extracts of growing cultures were analysed by LC/MS. (Solid line, no DMSO; dotted line, added DMSO).

The DMSO-induced effects were detected in both liquid and solid culture media; in the latter, the inclusion of DMSO often resulted in earlier and heavier sporulation. Other organic compounds were tested, but DMSO had the most dramatic effects. Dimethyl sulphone, a known metabolite of DMSO, had similar but less pronounced stimulatory effects.

In earlier studies with in vitro translation systems, DMSO was found to influence translation fidelity ${ }^{12)}$ (Gorini, L., and J. DAvies unpublished.). It was proposed that the increase in error frequency in cell-free systems also explained the ability of DMSO and ethanol to cause phenotypic suppression of auxotrophic mutants and promotion of the growth of antibiotic-dependent mutants in 
Fig. 5. Chromatographs of $S$. lividans TK23 carrying recombinant plasmids encoding DNA fragments obtained from soil DNA.
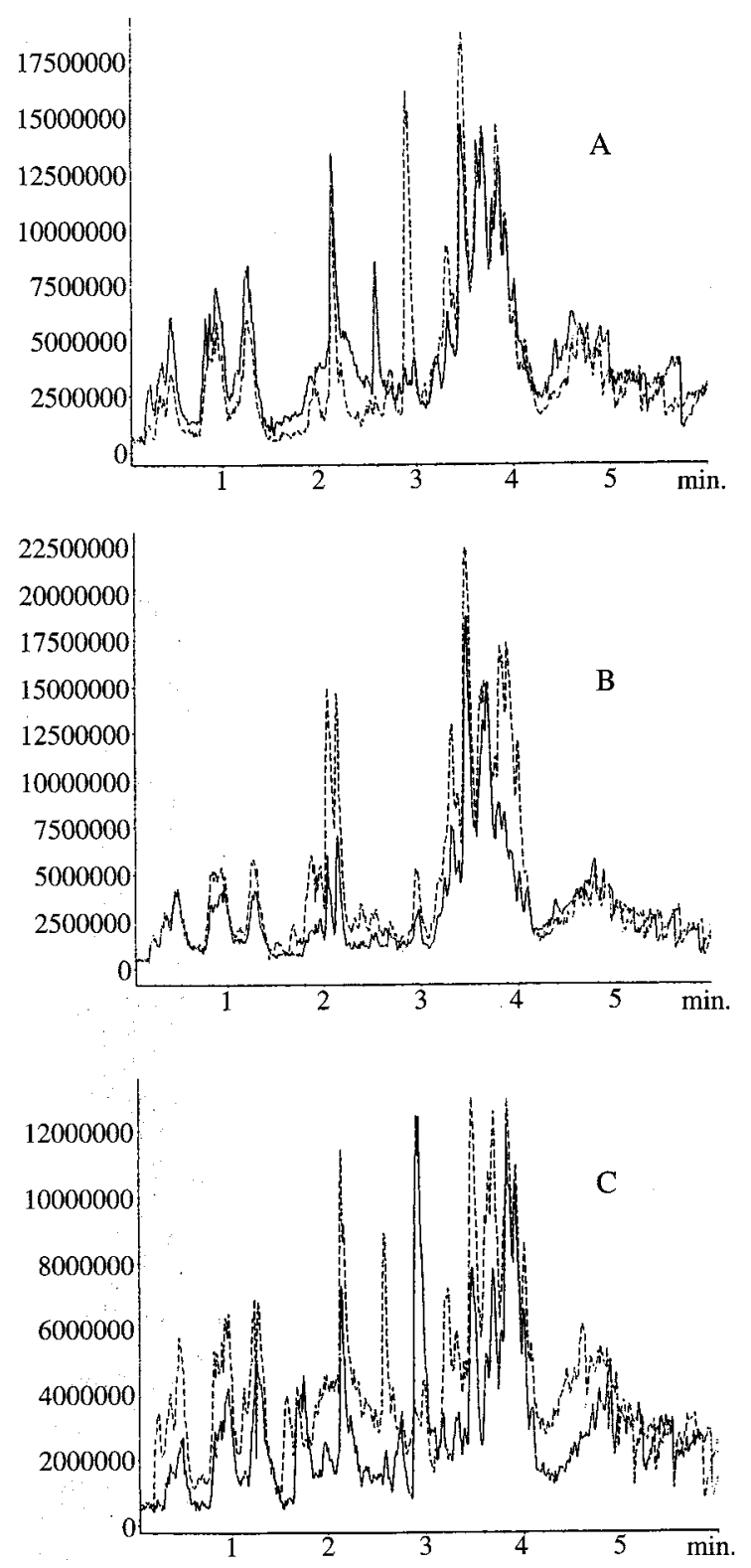

Solid line, no DMSO; dotted line, added DMSO.

(A) 446-S3-5-B12. (B) 446-S3-102H4. (C) 446-S381D3.

antibiotic-free medium. These effects have not been adequately explained, but it has been suggested that the organic compound causes subtle conformational changes (denaturation) of the ribosome or its components or possibly influences base pairing through tautomerisation of uracil residues ${ }^{12)}$. It is interesting to note that current understanding of the ribosome indicates that it is a dynamic structure that undergoes numerous conformational changes during the process of peptide bond formation ${ }^{22)}$. A solventinduced effect on any of these transitory active states may influence the translation process and so cause variations in the synthesis of effector molecules such as ppGpp, as reported by Hosoya et al. ${ }^{9)}$. However, we saw little or no change in concentration of this latter compound when DMSO was added to growing cultures (results not shown). The fact that EtOH stimulates tetracenomycin $\mathrm{C}$ but inhibits chloramphenicol production demonstrates that the effects of the solvent vary depending on the microbe and its physiology. Ethanol has long been known to cause phenotypic suppression of nonsense mutants in $E$. coli in the same way as $\mathrm{DMSO}^{23)}$, but it is not known if the two solvents act in the same way.

Recent studies by OCHI's group have shown that mutations to streptomycin resistance have marked effects on morphology and on the production of secondary metabolites ${ }^{8}$. For example, the introduction of certain streptomycin resistance alleles (rpsL) into $S$. lividans causes the bacterium to produce actinorhodin, a type II polyketide, which does not occur under normal growth conditions in this organism. In addition, SHIMA et al. reported that $S$. lividans grown in the presence of subinhibitory concentrations of streptomycin or tetracycline also produces actinorhodin ${ }^{11}$. Other studies by OCHI's group show that str alleles provoke antibiotic overproduction in other bacterial genera, including Streptomyces, Bacillus, and Pseudomonas. The active strR alleles affected ppGpp production in the host, and this combination of events was interpreted in terms of a causal interaction between the translation process and secondary metabolic events in streptomycetes.

Is there any relationship between the phenomena studied extensively by OCHI's group and the effects described herein with DMSO? As with strR, the effects of DMSO appear to be general and do not influence specific biosynthetic pathways. DMSO (an antioxidant) has been demonstrated to have various physiological effects ranging from analgesic, to anti-inflammatory, to antineoplasmic, and in addition it is widely used as a vehicle to promote drug penetration and delivery ${ }^{24)}$. Among its known cellular effects, DMSO recently has been shown to modulate nuclear factor-kappa $B$ and cytokine activation in lipopolysaccharide-treated macrophage ${ }^{25}$. These properties have been attributed to the dipolarity of $\mathrm{DMSO}^{26}$. However, whether this dipolar property plays a role in its effect on secondary metabolite production by microorganisms cannot be stated at this time. Chemicals with similar aprotic property, such as dimethylformamide 
and acetonitrile, did not influence the production of antibiotics such as chloramphenicol to the same extent as DMSO. Interestingly, dimethyl sulfone, an oxidised metabolite of DMSO, increased chloramphenicol production by $S$. venezuelae, although this was less pronounced. DMSO is a natural product that is made by many species of bacteria, especially anaerobes and organisms grown under low oxygen tension; it is present in significant concentrations in the atmosphere. Under anaerobic conditions DMSO is metabolised by the molybdoenzyme DMSO reductase as the terminal electron acceptor in microorganisms such as Rhodobacter spheroides $^{21,27)}$. DMSO has been shown to trigger a variety of phenotypic changes in Myxococcus xanthus ${ }^{28,29)}$.

Morphology and secondary metabolite production in bacteria $^{5)}$ and fungi ${ }^{30)}$ are changed when certain lowmolecular-weight compounds are present. In some cases, as with A-factor and a variety of other butyrolactones, they are naturally occurring microbial pheromones that are important effector molecules in the regulation of cellular differentiation and secondary metabolite production in streptomycetes $^{4)}$. Further work is needed to determine if DMSO and other organic solvents produce their effects on antibiotic production by mimicking the action of such pheromones; since DMSO is produced by many bacteria, it would not be surprising if it had a pheromone-like activity in nature. It is also possible that DMSO, like a number of antibiotics, acts through the induction of expression of specific classes of stress-proteins ${ }^{31)}$; this needs to be examined.

The practical implication of this work is that the addition of a small concentration of an organic compound (such as DMSO) as a component of the fermentation medium elicits physiological and biochemical changes in the growing cells, including secondary metabolite production. These studies do not distinguish between primary and secondary metabolites, and it is probable that the majority of biochemical pathways are affected by the presence of the organic compounds. This simple enrichment strategy could have value in expanding the available molecular diversity and increasing yields of minor metabolites from natural isolates or from genetically engineered recombinant strains.

\section{Acknowledgements}

We wish to thank DOROTHY DAVIES for preparing the manuscript and our TerraGen colleagues for their encouragement and technical help.

\section{References}

1) Hunt, G. R. \& R. W. Stieber: Inoculum development. In Manual of Industrial Microbiology and Biotechnology. Eds., A. L. Demain \& N. A. Solomon, pp. 32 48, American Society for Microbiology, Washington, DC, 1986

2) QueEner, S. W. \& D. H. Lively: Screening and selection for strain improvement. In Manual of Industrial Microbiology and Biotechnology. Eds., A. L. DEMAIN \& N. A. Solomon, pp. 155 169, American Society for Microbiology, Washington, DC, 1986

3) Süsstrunk, U.; J. Pidoux, S. Taubert, A. Ullmann \& C. J. ThOMPSON: Pleiotropic effects of cAMP on germination, antibiotic biosynthesis and morphological development in Streptomyces coelicolor. Mol. Microbiol. 30: 33 46, 1998

4) Kang, D.-K.; X.-M. Li, K. OCHI \& S. Horinouchi: Possible involvement of cAMP in aerial mycelium formation and secondary metabolism in Streptomyces griseus. Microbiology 145: 1161 1172, 1999

5) BEPPU, T.: Signal transduction and secondary metabolism: prospects for controlling productivity. Trends Biotechnol. 13: 264 269, 1995

6) BiBb, M.: The regulation of antibiotic production in Streptomyces coelicolor A3(2). Microbiology 142: 1335 1344, 1996

7) BALtz, R. H.: Molecular genetic approaches to yield improvement in actinomycetes. In Biotechnology of Antibiotics. Ed., W. R. STROHL, pp. 49 62, Marcel Dekker, New York, 1997

8) Hesketh, A. \& K. OCHI: A novel method for improving Streptomyces coelicolor A3(2) for production of actinorhodin by introduction of $r p s L$ (encoding ribosomal protein $\mathrm{S} 12$ ) mutations conferring resistance to streptomycin. J. Antibiotics 50: 532 535, 1997

9) Hosoya, Y.; S. Okamoto, H. Muramatsu \& K. OChI: Acquisition of certain streptomycin-resistant (str) mutations enhances antibiotic production in bacteria. Antimicrob. Agents Chemother. 42: 2041 2047, 1998

10) Holt, T. G.; C. Chang, C. Laurent-Winter, T. Murakami, J. I. Garrels, J. E. Davies \& C. J. THOMPSON: Global changes in gene expression related to antibiotic biosynthesis in Streptomyces hygroscopicus. Mol. Microbiol. 6: 969 980, 1992

11) Shima, J.; A. Hesketh, S. OKamoto, S. Kawamoto \& K. OCHI: Induction of actinorhodin production by $r p s L$ (encoding ribosomal protein S12) mutations that confer streptomycin resistance in Streptomyces lividans and Streptomyces coelicolor A3(2). J. Bacteriol. 178: 7276 7284,1996

12) Gorini, L. \& J. Davies: The effect of streptomycin in ribosomal function. Curr. Top. Microbiol. Immunol. 44: $100 \sim 122,1968$

13) So, A. G. \& E. W. Davie: The effects of organic solvents on protein biosynthesis and their influence on the amino acid code. Biochemistry 3: 1165 1169, 1964

14) So, A. G. \& E. W. DaviE: Effects of amino acids, sRNA, and ethanol on coding ambiguity with polyuridylic acid. Biochemistry 4: 1973 1979, 1965

15) GORIni, L. \& E. KaTAJA: Phenotypic repair by streptomycin of defective genotypes in E. coli K-12. 
Proc. Natl. Acad. Sci. USA 51: 487 493, 1964

16) Hopwood, D. A.; M. J. BibB, K. F. Chater, T. Kieser, C. J. Bruton, H. M. Kieser, D. J. Lydiate, C. P. Smith, J. M. WARD \& H. SCHREMPF: Genetic Manipulation of Streptomyces: a Laboratory Manual. The John Innes Foundation, Norwich, UK, 1985

17) Lazar, G.; H. Zahner, S. Breiding, M. Damberg \& A. ZEECK: 3-Dimethoxyl-3-ethoxy-tetracenomycin C. J. Antibiotics 34: 1067 1068, 1981

18) MaliK, V. S. \& L. C. Vining: Metabolism of chloramphenicol by the producing organism. Can. J. Microbiol. 16: 173 180, 1970

19) Bierman, M.; R. Logan, K. O'brien, E. T. Seno, R. Rao \& B. E. SCHONER: Plasmid cloning vectors for the conjugal transfer of DNA from Escherichia coli to Streptomyces spp. Gene 116: 43 49, 1992

20) Guilfoile, P. G. \& C. R. Hutchinson: A bacterial analog of the $m d r$ gene of mammalian tumor cells is present in Streptomyces peucetius, the producer of daunorubicin and doxorubicin. Proc. Natl. Acad. Sci. USA 88: 8553 8557, 1991

21) Mouncey, N. J.; M. Choudhary \& S. Kaplan: Characterization of genes encoding dimethyl sulfoxide reductase of Rhodobacter sphaeroides $2.4 .1^{\mathrm{T}}$ : an essential metabolic gene function encoded on chromosome II. J. Bacteriol. 179: 7617 7624, 1997

22) Cate, J. H.; M. M. Yusupov, G. Z. Yusupova, T. N. EARNEST \& H. F. NOLLER: X-ray crystal structures of 70S ribosome functional complexes. Science 285: 2095 2104, 1999

23) So, A. G.; J. W. Bodley \& E. W. Davie: The influence of environment on the specificity of polynucleotide dependent amino acid incorporation into polypeptide. Biochemistry 3: 1977 1982, 1964

24) JACOB, S. W. \& R. HeRSCHLER: Pharmacology of DMSO. Cryobiology 23: 14 27, 1986

25) Kelly, K. A.; M. R. Hill, K. Youkhana, F. Wanker \& J. M. GimBLE: Dimethyl sulfoxide modulates NF-kappa $\mathrm{B}$ and cytokine activation in lipopolysaccharide-treated murine macrophages. Infect. Immun. 62: 3122 3128, 1994

26) Kharasch, N. \& B. S. Thyagarajan: Structural basis for biological activities of dimethyl sulfoxide. Ann. N.Y. Acad. Sci. 411: 391 402, 1983

27) Mouncey, N. J. \& S. Kaplan: Redox-dependent gene regulation in Rhodobacter sphaeroides $2.4 .1^{\mathrm{T}}$ : effects on dimethyl sulfoxide reductase (dor) gene expression. J. Bacteriol. 180: 5612 5618, 1998

28) Komano, T.; S. Inouye \& M. Inouye: Patterns of protein production in Myxococcus xanthus during spore formation induced by glycerol, dimethyl sulfoxide, and phenethyl alcohol. J. Bacteriol. 144: 1076 1082, 1980

29) SHI, W.; T. KOHLER \& D. R. ZuSMAN: Isolation and phenotypic characterization of Myxococcus xanthus mutants which are defective in sensing negative stimuli. J. Bacteriol. 176: 696 701, 1994

30) Schimmel, T. G.; A. D. Coffman \& S. J. Prsons: Effect of butyrolactone I on the producing fungus Aspergillus terreus. Appl: Environ. Microbiol. 64: 3707 3712, 1998

31) VanBogelen, R. A. \& F. C. Neidhardt: Ribosomes as sensors of heat and cold shock in Escherichia coli. Proc. Natl. Acad. Sci. USA 87: 5589 5593, 1990 